\title{
Prolonged Vertigo Associated with Nystagmus: an Organic or a Psychiatric Disorder? Discussing a Case Report
}

\author{
Naeimehossadat Hosseini ${ }^{1}$, Amir Moghadam-Ahmadi² ${ }^{2}$ Mohammad Ali Abdolkarimi Dawarani ${ }^{3}$, Reza Bidaki ${ }^{4}$, \\ Aboozar Tajiksafa ${ }^{5}$
}

${ }^{1}$ Isfahan medical student research committee, Isfahan University of Medical Sciences, Isfahan, Iran

${ }^{2} \mathrm{Head}$ of clinical research development center, Rafsanjan University of Medical Sciences, Rafsanjan, Iran

${ }^{3}$ Rafsanjan University of Medical Sciences, Rafsanjan, Iran

${ }^{4}$ Rafsanjan University of Medical Sciences, Rafsanjan, Iran

${ }^{5}$ Rafsanjan University of Medical Sciences, Rafsanjan, Iran

\begin{abstract}
Background:It is mostly organic diseases that are considered when a neurological sign or symptom is present in the history of a patient; however, there may be psychiatric problems also present which could be missed. We report a case to discuss the possibility of the presence of an organic or psychiatric underlying disorder when facing neurological problems.Case Presentation:We report a case of chronic headache accompanied by vertigo and multiple medical problems including nystagmus and abdominal pain without any significant findings on physical exam that was diagnosed to have a somatization disorder (Not Otherwise Specified or NOS).Conclusion:We did not find any documents regarding the presence of true nystagmus as a functional, factitious or psychosomatic disorder; however, voluntary nystagmus has been reported in some cases with a series of fast (saccadic) back-to-back eye movements, without any interval or slow phase. It is recommended that psychiatrists and neurologists search for a psychiatric disorder as well; even if the problem is supposed to have an organic nature like nystagmus or vertigo, especially when associated with a prolonged, resistant, or unusual course.
\end{abstract} [GMJ. 2013;2(2):83-86]

Keywords:Nystagmus; Vertigo; Somatization disorder

\section{Introduction}

Vertigo, known as an illusion of movelogic associated complaints in medicine [1,2]. It can be defined as subjective (abnormal sensation of movement with person's body) or objective (the environment) [3]. Vertigo is classified according to duration of symptoms and the condition causing the symptoms,

\section{GMJ}

2013 Galen Medical Journal

Fax: +98 7312227091

PO Box 7461686688

Email:info@gmj.ir whether it is positional or starts spontaneously. Also presence of hearing loss or tinnitus can provide important clues in identifying the cause of vertigo [3]. Vertigo can result from a psychological condition [3] as well as causing psychological consequences such as interfering with one's social life, anxiety, depression, and panic disorder. Vertigo is generally accompanied by nystagmus [4]. Nystagmus is a

\footnotetext{
Correspondence to:

Reza Bidaki,Psychiatry service, Moradi hospital,

Rafsanjan, Iran

Telephone Number:+983915230081

Email Address :-
} 
common clinical condition, described as fast involuntary repetitive eye movements which might be from side to side (horizontal) up and down (vertical) or torsional. Conditions associated with nystagmus can be congenital, such as cataract, congenital eye defects, acquired, such as medications, vitamin deficiency, vestibular problems, trauma, stroke and brain tumors, or physiological [5]. According to the Fourth Edition Text Revision of The diagnostic and Statistical Manual of Mental Disorders (DSM-IV-TR)[6], somatoform disorders is a group of disorders characterized by the presence of physical signs without organic cause on medical evaluations. The most common group of somatoform disorders seen in clinical practice is somatization disorder which has special characteristics such as onset of unexplained medical symptoms before the age of 30 years old, multiple chronic complaints of unexplained physical symptoms, multiple pain symptoms, two or more gastrointestinal symptoms and at least one pseudo-neurological symptom [7].

\section{Case Presentation}

A 48-year-old female presented with chief complaint of headache. The headache was bilateral, compressing on the parietal region. She had been having the headaches since about 2.5 years ago; while she had no other complaints. The patient had a positive history of hypertension, hyperlipidemia, and hypothyroidism for which she was receiving Amlodipine 5mg/day and Levothyroxine 0.1 $\mathrm{mg} /$ day. Her general physical examination and neurologic examination, vital signs, and laboratory tests including complete blood count, urinalysis, and chemistry profile were all within normal limits. It was first suggested that her headache was secondary to high blood pressure. Regarding the persistence of headache even after appropriate control of hypertension, a neurologist decided to perform Computed Tomography (CT) scan and then Magnetic Resonance Imaging (MRI) in order to rule out intracranial lesions. CT scan and MRI did not reveal significant findings and she was diagnosed to have tension headaches. Based on these findings, the patient was dis- charged with proper medications including Sodium-Valproate (Orlept), Topiramate, and Gabapentine.

During a 2-months period follow-up, headaches did not alleviate and episodes of vertigo were added to previous complaints. The vertigo attacks were positional, lasting more than 1 hour each time. She had no associated nausea or vomiting at that time. Other findings on physical exam were all normal. In order to evaluate the cause of vertigo, with suspicion to vestibular diseases such as vestibular neuritis or Menier's disease, audiometry was performed. Normal results in all components of the audiometery tests, Dix-Hallpike maneuver, and Caloric test implied vestibulitis. She was discharged with administration of Prednisolone $0.1 \mathrm{mg}$ daily. She developed diabetes mellitus while receiving Prednisolone and her symptoms did not alleviate despite the therapy. Prednisolone was tapered and she was subjected to insulin therapy due to her newonset diabetes. She received Acetazolamide $250 \mathrm{mg}$ twice per day for two weeks due to the possibility of high intra-cranial pressure but it failed to reduce her complaints of pain, and therefore was discontinued. During follow up, she developed vomiting in addition to vertigo. On physical examination, she had a persistent vertical nystagmus which raised the suspicion for presence of an organic cause. The patient also developed balance disturbances (ataxia) and could not walk without aid. She was then sent to a specialty clinic for neurological evaluations that did not reveal any abnormalities. Imaging and physical exams were normal. Furthermore, the patient started to suffer from panic attacks and generalized body tremor. She also complained of excessive sweating that was worse in the evening. Tremors were accompanied by abdominal pain, rigidity and itchy sensation in the shoulder girdle. However, the patient reported that the symptoms were relieved when lying in a prone position. Her physical examinations remained insignificant for any intra-abdominal pathology. For further work up, she was referred to a psychiatrist where she was diagnosed to have conversion disorder (Mixed type). She was then hospitalized and treated with 8 sessions of Electroconvulsive therapy (ECT); howev- 
er, no substantial improvement was observed in patient's condition. After six months of significant disability due to persistent nausea and vomiting, vertigo, and abdominal pain, she was referred to a psychiatry center where she was diagnosed with somatization disorder in accordance with DSM-IV. She spent 15 days in the psychiatry ward. She was discharged with Doxepine, Zolpidem, Quetiapine, Alprazolam, and Venlafaxine. Within one month, all of her symptoms subsided. Although she still complained of infrequent episodes of emesis.

\section{Discussion}

We have reported a patient with chronic headache accompanied by vertigo and multiple medical problems including nystagmus, and abdominal pain. The patient had no significant finding on physical examination and had been diagnosed to have a somatization disorder (Not Otherwise Specified or NOS).

Psychogenic ocular disorders can represent up to $5 \%$ of an ophthalmologist's practice. They include functional disease, psychosomatic disease, and artificial (or factitious) eye diseases [8]. In this study, we reported a case in which nystagmus with vertigo did not have concordance with any organic disorders.

Nonorganic (functional) visual disturbances are subjectively described visual disorders without an objectively observed abnormality on examination. They can have a variety of manifestations such as visual acuity loss, visual field loss, color perception abnormalities, diplopia, night blindness, voluntary nystagmus, etc. Providing a challenge to diagnosis and management like other nonorganic conditions our case had no visual disturbance or ocular finding.

A careful social and family history must be obtained when suspecting a nonorganic visual disturbance, not only on the basis of the patient's behavior, but also with a poorly described visual complaint without any apparent morphological correlates. Moreover, organic ocular and visual disorders that can present without related physical findings must be ruled out of the differential diagnosis. It is of utmost importance to keep in mind that the diagnosis of functional or simulated visual loss cannot be made purely as a diagnosis of exclusion, and positive findings and specific malingering tests are necessary in order to make an accurate diagnosis [8].

Different examination approaches are necessary for different nonorganic visual disturbances, such as examining the pupils and checking for optokinetic nystagmus in visual field testing for a patient with suspected nonorganic visual loss $[8,9]$. Factitious abnormalities in the visual-evoked potentials (VEPs) can be induced by normal persons who defocus their vision during the test; therefore, the use of VEP to diagnose nonorganic visual loss can be frustrating and unreliable. If the VEP is normal, useful information is gained; however, an abnormal VEP is not always diagnostic of an organic visual disturbance [9]. According to the previous history and assessing, it was not achieved any facticious disorder (any primary gain with sick role) or malingering (any secondary gain).

Overall, many patients with nonorganic visual disturbance have a good prognosis and at least half of them improve with time and reassurance. Factors that indicate a poor prognosis include older age and depression, whereas youth and the presence of anxiety are usually associated with a good prognosis $[8,9]$.

Psychosomatic eye disease is initiated by an organic disease with demonstrable pathological findings that is psychically triggered or heavily influenced. Artificial eye diseases arise by self-inflicted trauma (auto aggression) and have demonstrable pathological findings on examination [8].

We did not find any documents regarding the presence of true nystagmus as a functional, factitious or psychosomatic disorder; however, voluntary nystagmus has been reported in some cases with a series of fast (saccadic) back-to-back eye movements, without any interval or slow phase. Ocular flutter is not a true nystagmus, can be created under voluntary control, and is usually horizontal but may be vertical, torsional, or (rarely) cycloid. Usually, persons with this ability, which is a familial problem in some instances, must converge their eyes to initiate the oscillation. Occasionally, patients use this ability to feign illness; however, it can be recognized easily 
as it cannot be sustained longer than 30 seconds [9].

Functional or factitious disorders do not frequently present with true vertigo. Most of the patients with these problems probably have nonspecific dizziness rather than true vertigo and they are usually bothered by lightheadedness (wooziness), pre-syncope, imbalance, motion sensitivity, or anxiety. Nonspecific dizziness can present secondary to cardiac arrhythmias or metabolic disturbances or as a side effect or toxicity from medications. Similarly, bothersome lightheadedness can be a direct effect of the medication itself or the result of lowering of the patient's blood pressure or even dehydration or vasovagal attacks. Since many elderly patients are taking multiple drugs including blood pressure medications, at least a trial of lowering or discontinuing these medications is recommended in such patients with a nonspecific dizziness.
Nonspecific dizziness can also be one of the features of panic attacks, but these spells are invariably accompanied by other symptoms such as sense of fear or doom, palpitations, sweating, shortness of breath, or paresthesia. Among these, dizziness might disappear by appropriate treatment of panic attack [9].

Conclusion

Some advocate considering vertigo "psychiatric" only if it is part of a recognized psychiatric symptom complex and cannot be explained by an organic disease. The importance of this case report is to recommend that physicians consider both psychiatric and organic causes for the neurologic problems that appear to have solely an organic nature; especially when the problem has a prolonged or refractory course.

\section{Declaration of interest: None}

\section{References}

1. Gilain C, Englebert A. Vertigo and psychological disorders. B-ENT. 2008;4(8):49 -58.

2. Hain TC, Yacovino D. Pharmacologic treatment of persons with dizziness. Neurol Clin. 2005;23(3):831-53.

3. Strupp M, Brandt T. Diagnosis and treatment of vertigo and dizziness. Dtsch Arztebl Int. 2008;105(10):173-80.

4. Warner EA, Wallach PM, Adelman HM, Sahlin-Hughes K. Dizziness in primary care patients. J Gen Intern Med. 1992;7(4):454-63.

5. Abadi RV. Mechanisms underlying nystagmus. J R Soc Med. 2002;95(5):2314.
6. Association AP. Diagnostic and statistical manual of mental disorders: DSM-IV-TR: American Psychiatric Publishing, Inc; 2000.

7. Kallivayalil RA, Punnoose VP. Understanding and managing somatoform disorders: Making sense of non-sense. Indian J Psychiatry. 2010; 52(1):240-5.

8. Schiefer U, Wilhelm H, Hart W. Clinical Neuro-Ophthalmology: A Practical Guide. Berlin Heidelgberg: Springer-Verlag;2007. p.203-14.

9. Daroff RB, Fenichel GM, Jankovic J, Mazziotta JC. Bradley's Neurology in Clinical Practice. $6^{\text {th }}$ ed: Elsevier; 2012. Vol 1, p. 609, 621-2, 657 . 